\title{
Effect of organic acids on the growth and lipid accumulation of oleaginous yeast Trichosporon fermentans
}

\author{
Chao Huang ${ }^{1}$, Hong Wu' ${ }^{1 *}$, Zong-jun Liu², Jun Cai ${ }^{3}$, Wen-yong Lou' and Min-hua Zong ${ }^{1 *}$
}

\begin{abstract}
Background: Microbial lipids have drawn increasing attention in recent years as promising raw materials for biodiesel production, and the use of lignocellulosic hydrolysates as carbon sources seems to be a feasible strategy for cost-effective lipid fermentation with oleaginous microorganisms on a large scale. During the hydrolysis of lignocellulosic materials with dilute acid, however, various kinds of inhibitors, especially large amounts of organic acids, will be produced, which substantially decrease the fermentability of lignocellulosic hydrolysates. To overcome the inhibitory effects of organic acids, it is critical to understand their impact on the growth and lipid accumulation of oleaginous microorganisms.
\end{abstract}

Results: In our present work, we investigated for the first time the effect of ten representative organic acids in lignocellulosic hydrolysates on the growth and lipid accumulation of oleaginous yeast Trichosporon fermentans cells. In contrast to previous reports, we found that the toxicity of the organic acids to the cells was not directly related to their hydrophobicity. It is worth noting that most organic acids tested were less toxic than aldehydes to the cells, and some could even stimulate the growth and lipid accumulation at a low concentration. Unlike aldehydes, most binary combinations of organic acids exerted no synergistic inhibitory effects on lipid production. The presence of organic acids decelerated the consumption of glucose, whereas it influenced the utilization of xylose in a different and complicated way. In addition, all the organic acids tested, except furoic acid, inhibited the malic activity of $T$. fermentans. Furthermore, the inhibition of organic acids on cell growth was dependent more on inoculum size, temperature and initial $\mathrm{pH}$ than on lipid content.

Conclusions: This work provides some meaningful information about the effect of organic acid in lignocellulosic hydrolysates on the lipid production of oleaginous yeast, which is helpful for optimization of biomass hydrolysis processes, detoxified pretreatment of hydrolysates and lipid production using lignocellulosic materials.

Keywords: lignocellulosic hydrolysate, organic acid, inhibition, lipid production, Trichosporon fermentans

\section{Background}

Biodiesel, a mixture of long-chain monoalkyl fatty acid esters, has been considered a good alternative to conventional petrodiesel oil because of its green and renewable characteristics [1]. Although it has been used in many countries around the world, the high production cost, of which oil feedstock accounts for about 75\%, has become a hurdle, and the sustainable and stable supply of cheap

\footnotetext{
* Correspondence: bbhwu@scut.edu.cn; btmhzong@scut.edu.cn 'State Key Laboratory of Pulp and Paper Engineering, College of Light Industry and Food Sciences, South China University of Technology, 381 Wushan Rd.Tianhe District, Guangzhou 510640, China Full list of author information is available at the end of the article
}

lipids is crucial for their further development and wide application [2]. Nowadays, the most commonly used feedstocks in biodiesel production are vegetable oils and waste oils from restaurants or industry. However, vegetable oils such as rapeseed oil and corn oil contribute to the world's food supply, and thus their use as feedstock for biodiesel production has brought about the food versus biofuel debate [3]. The amount of waste oils is limited and cannot meet the increasing demand for biofuel. Microbial oils, namely, single-cell oils (SCOs), which have long been used as substitutes for high-added-value lipids $[4,5]$ such as cocoa butter [6,7], are now believed to be a promising
C Biomed Central 
candidate as biodiesel feedstock because of their fatty acid composition, which is similar to those of vegetable oils [8]. At present, however, the high fermentation cost of SCOs limits their use for biodiesel production $[7,9]$. The adoption of inexpensive media, such as molasses [10], industrial glycerol [11], monosodium glutamate wastewater [2] and lignocellulosic hydrolysates [12] for lipid fermentation is one of the possible resolutions of this problem. Recently, the use of lignocellulosic materials for SCO production has attracted increasing attention because these materials are the most abundant and renewable biomass resources in nature $[8,12]$

Lignocellulosic biomass consists of cellulose, hemicellulose and lignin, whose relative proportion depends on their material sources [13]. The hydrolysis of lignocellulosic materials into soluble, fermentable sugars is necessary for their efficient utilization by microorganisms. However, a variety of by-products, mainly organic acids, aldehydes and alcohols such as acetic acid, furfural from decomposition of pentoses, 5-hydroxymethylfurfural from degradation of hexoses and aromatics (aromatic alcohols, acids and aldehydes) from lignin, are inevitably generated during hydrolysis with dilute acid [14]. In most cases, these byproducts, known as "inhibitors," exert negative effects on the growth, metabolism and product formation of microorganism cells in the fermentation process [15].

Recently, we reported that despite the oleaginous yeast Trichosporon fermentans's production of a poor lipid yield on nondetoxified, sulfuric acid-treated rice straw hydrolysate (SARSH), it grew well with efficient lipid accumulation on detoxified SARSH [12], suggesting that the inhibitors in the lignocellulosic hydrolysate do have great effects on lipid fermentation. Among the inhibitors, organic acids are generally the most abundant, and ten kinds of organic acids, including aliphatic acids (acetic acid, formic acid, levulinic acid and caproic acid), aromatic or furan acids (4-hydroxybenzoic acid, syringic acid, vanillic acid, furoic acid, ferulic acid and gallic acid) have been found in lignocellulosic hydrolysate. Little is known about their inhibition on lipid fermentation, however [16,17]. To provide some interesting information necessary for lipid fermentation on lignocellulosic hydrolysates, we systematically investigated, for the first time, the inhibitory effects of the above-mentioned organic acids on the growth and lipid accumulation of T. fermentans with a mixture of glucose and xylose at a ratio of 2:1 (wt/wt) as the carbon source, owing to its similarity to lignocellulosic hydrolysates.

\section{Results and discussion}

All microbial processes are affected by the sugar concentration in the medium, and substrate inhibition may occur during growth of oleaginous microorganisms on sugars [18]. Therefore, we first investigated the effect of

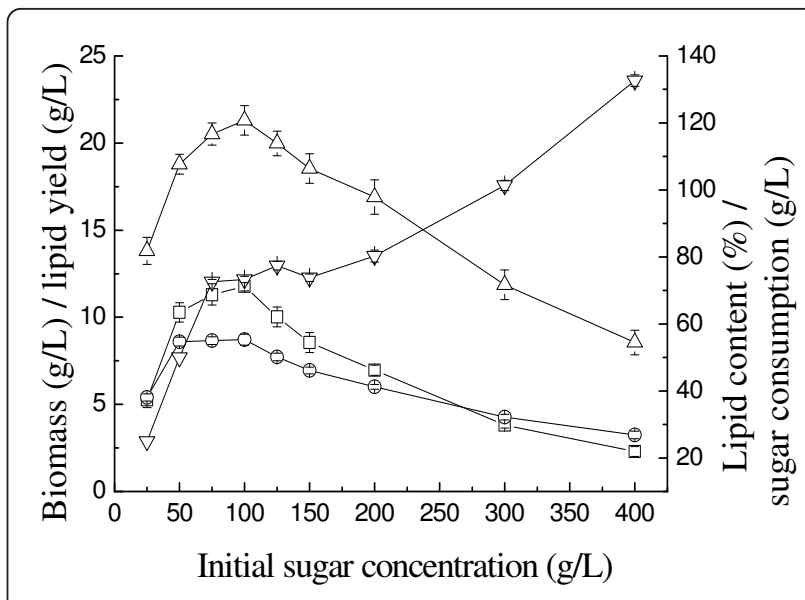

Figure 1 Effect of initial sugar concentration on the growth and lipid accumulation of Trichosporon fermentans. Triangles, biomass; circles, lipid content; squares, lipid yield; inverted triangles, sugar consumption.

sugar concentration, ranging from 25 to $400 \mathrm{~g} / \mathrm{L}$, on cell growth, lipid accumulation and sugar consumption of $T$. fermentans. As shown in Figure 1, there was no significant substrate inhibition on the cell growth of T. fermentans at concentrations up to $100 \mathrm{~g} / \mathrm{L}$, but cell growth was repressed at higher sugar concentrations, especially $\geq 200$ $\mathrm{g} / \mathrm{L}$. The highest biomass, lipid content and lipid yield occurred at $100 \mathrm{~g} / \mathrm{L}$ sugar concentration. At this point, about $73.3 \mathrm{~g} / \mathrm{L}$ total sugars were utilized by $T$. fermentans after five days' fermentation, and the biomass and lipid yield per sugar consumed were $29.1 \%$ and $16.1 \%(\mathrm{~g} / \mathrm{g})$, respectively. Therefore, $100 \mathrm{~g} / \mathrm{L}$ was chosen as the initial sugar concentration in the subsequent experiments.

In our previous study, we showed that the optimal inoculum size, temperature and initial $\mathrm{pH}$ for lipid production by $T$. fermentans at $100 \mathrm{~g} / \mathrm{L}$ glucose are $5 \%, 25^{\circ} \mathrm{C}$ and 6.5, respectively [10]. In our present work, albeit using a mixture of glucose and xylose $(100 \mathrm{~g} / \mathrm{L})$ at a ratio of $2: 1$ (wt/wt) as a carbon source, there was no change in the optimal fermentation conditions (inoculum size, temperature and initial $\mathrm{pH}$ ), as indicated in Table 1 . Figure 2 depicts the time courses of cell growth, lipid accumulation and sugar utilization of T. fermentans in the same medium against fermentation time. As shown in Figure $2 \mathrm{~A}$, the biomass increased with the increase of fermentation time and reached the maximum on day 7. Further increase in the fermentation time resulted in little variation in biomass production. The highest lipid content and lipid yield were also obtained on the seventh day, on which the SCO produced per total sugar consumed was $17.6 \%(\mathrm{~g} / \mathrm{g})$. Interestingly, $T$. fermentans could utilize glucose and xylose simultaneously (Figure 2B), which would be beneficial for large-scale application because it would shorten the fermentation time. 
Table 1 Effect of fermentation conditions on the cell growth and lipid accumulation of Trichosporon fermentans on the medium without inhibitor

\begin{tabular}{lccccc}
\hline $\begin{array}{l}\text { Inoculum size } \\
(\%, \text { vol/vol) }\end{array}$ & Initial $\mathbf{~ H H}$ & $\begin{array}{c}\text { Temperature } \\
\left({ }^{\circ} \mathbf{C}\right)\end{array}$ & Biomass $(\mathbf{g} / \mathbf{L})$ & $\begin{array}{c}\text { Lipid content } \\
(\%, \mathbf{g} / \mathbf{g})\end{array}$ & Lipid yield (g/L) \\
\hline $5 \%$ & 6.5 & 25 & $24.0 \pm 0.7$ & $61.7 \pm 1.6$ & $14.8 \pm 0.5$ \\
$10 \%$ & 6.5 & 25 & $22.4 \pm 1.2$ & $58.6 \pm 1.7$ & $13.1 \pm 1.0$ \\
$15 \%$ & 6.5 & 25 & $21.6 \pm 1.1$ & $54.3 \pm 1.2$ & $11.7 \pm 0.9$ \\
$5 \%$ & 5.5 & 25 & $18.4 \pm 0.9$ & $57.2 \pm 1.7$ & $10.5 \pm 0.8$ \\
$5 \%$ & 7.5 & 25 & $21.5 \pm 0.7$ & $56.3 \pm 1.4$ & $12.1 \pm 0.7$ \\
$5 \%$ & 6.5 & 22 & $19.9 \pm 0.9$ & $55.8 \pm 1.1$ & $11.1 \pm 0.7$ \\
$5 \%$ & 6.5 & 28 & $23.6 \pm 1.2$ & $58.9 \pm 1.2$ & $13.9 \pm 1.0$ \\
\hline
\end{tabular}

Effects of organic acids on the growth and lipid accumulation of $T$. fermentans

Among the ten kinds of acids tested, caproic and ferulic acids showed the strongest inhibitory effects, and the relative biomass levels of $T$. fermentans were only about $3 \%$ and $23 \%$ in the presence of $17.2 \mathrm{mM}(2 \mathrm{~g} / \mathrm{L})$ caproic

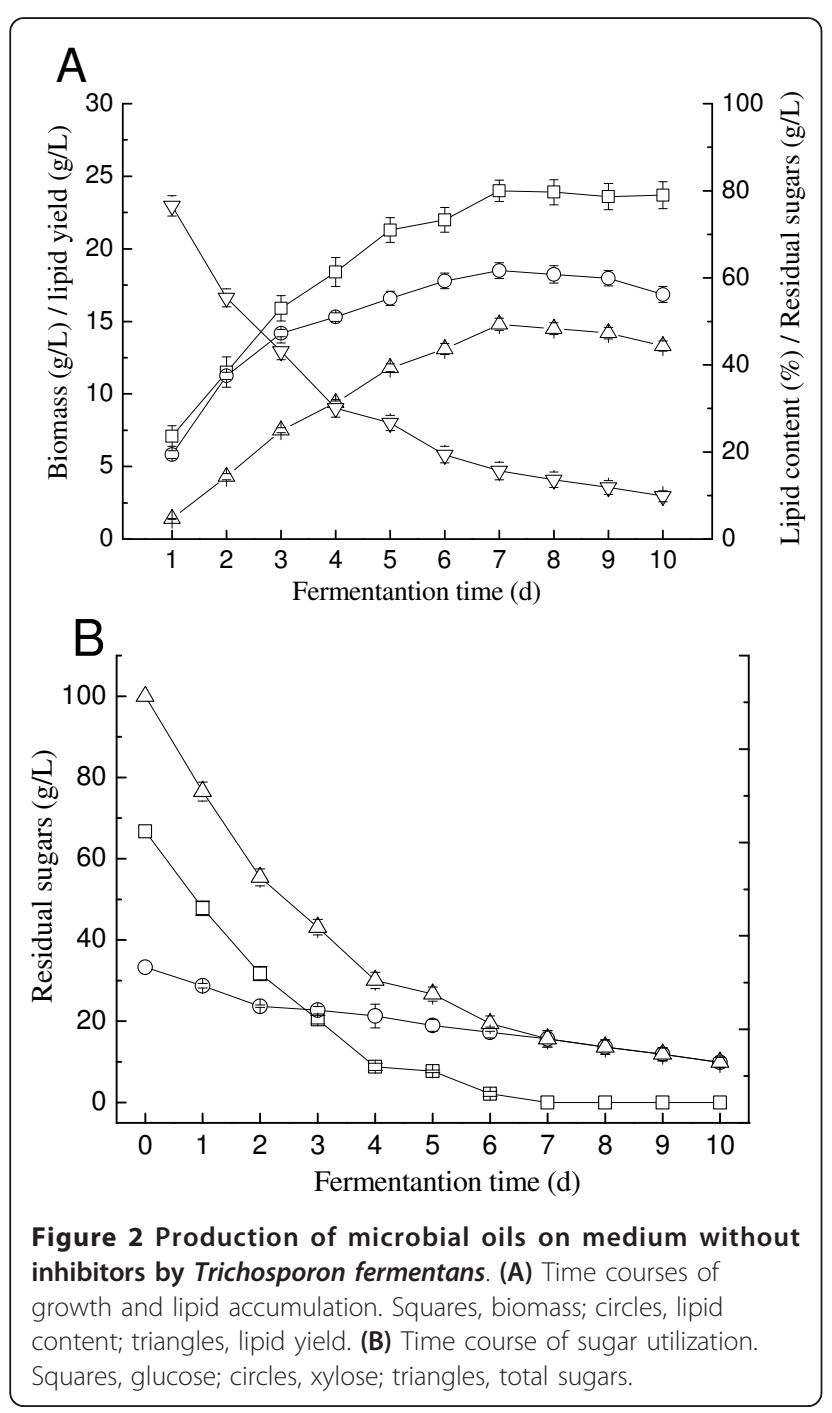

acid and $20.6 \mathrm{mM}(4 \mathrm{~g} / \mathrm{L})$ ferulic acid, respectively (Figure 3). Fortunately, caproic and ferulic acids are normally found at very low concentrations in lignocellulosic hydrolysates. For example, in brewer's spent grain and red oak hydrolysates, the concentrations of ferulic acid and caproic acid are only around $0.3 \mathrm{mM}(0.06 \mathrm{~g} / \mathrm{L})$ and $0.17 \mathrm{mM}(0.02 \mathrm{~g} / \mathrm{L})$, respectively $[19,20]$, which cause about $4 \%$ and $2 \%$ inhibition of lipid yield of $T$. fermentans, respectively (Figure 3), and hardly affect the lipid production by $T$. fermentans. The inhibitory effects of the other eight kinds of acids are shown in Figure 3. Similarly, all these organic acids showed only a slight inhibitory effect on the growth and lipid accumulation of $T$. fermentans at their likely concentrations in lignocellulosic hydrolysates [15]. Interestingly, although the inhibition of furoic acid on cell growth became more serious with the increase of its concentration, no significant decrease in the lipid content was observed, even when its concentration reached $89.2 \mathrm{mM}(10 \mathrm{~g} / \mathrm{L})$, which differs from previous reports that both cell growth and lipid synthesis of oleaginous yeast Rhodosporidium toruloides were seriously suppressed by furoic acid, even at concentrations as low as $4 \mathrm{mM}$ [17], demonstrating that the inhibition of an organic acid on lipid fermentation varies widely with different microorganism strains. Among the organic acids tested, levulinic acid exerted the least impact on the lipid yield. It is worth noting that, rather than suppressing cell growth and lipid accumulation, some organic acids, including formic acid, acetic acid, levulinic acid, 4-hydroxybenzoic acid and gallic acid, might even stimulate both cell growth and lipid accumulation of $T$. fermentans when their concentrations are below $16 \mathrm{mM}$. A similar phenomenon was also observed in that low concentrations of phenol-type compounds $(<5 \mathrm{mM}$ of gallic acid equivalent) stimulated biomass production and citric acid biosynthesis of yeast Yarrowia lipolytica when olive mill waste waters were used as a substrate for citric acid production [21]. Also, it has been reported that the biomass and SCO production by another $Y$. lipolytica strain was remarkably enhanced when the medium was 


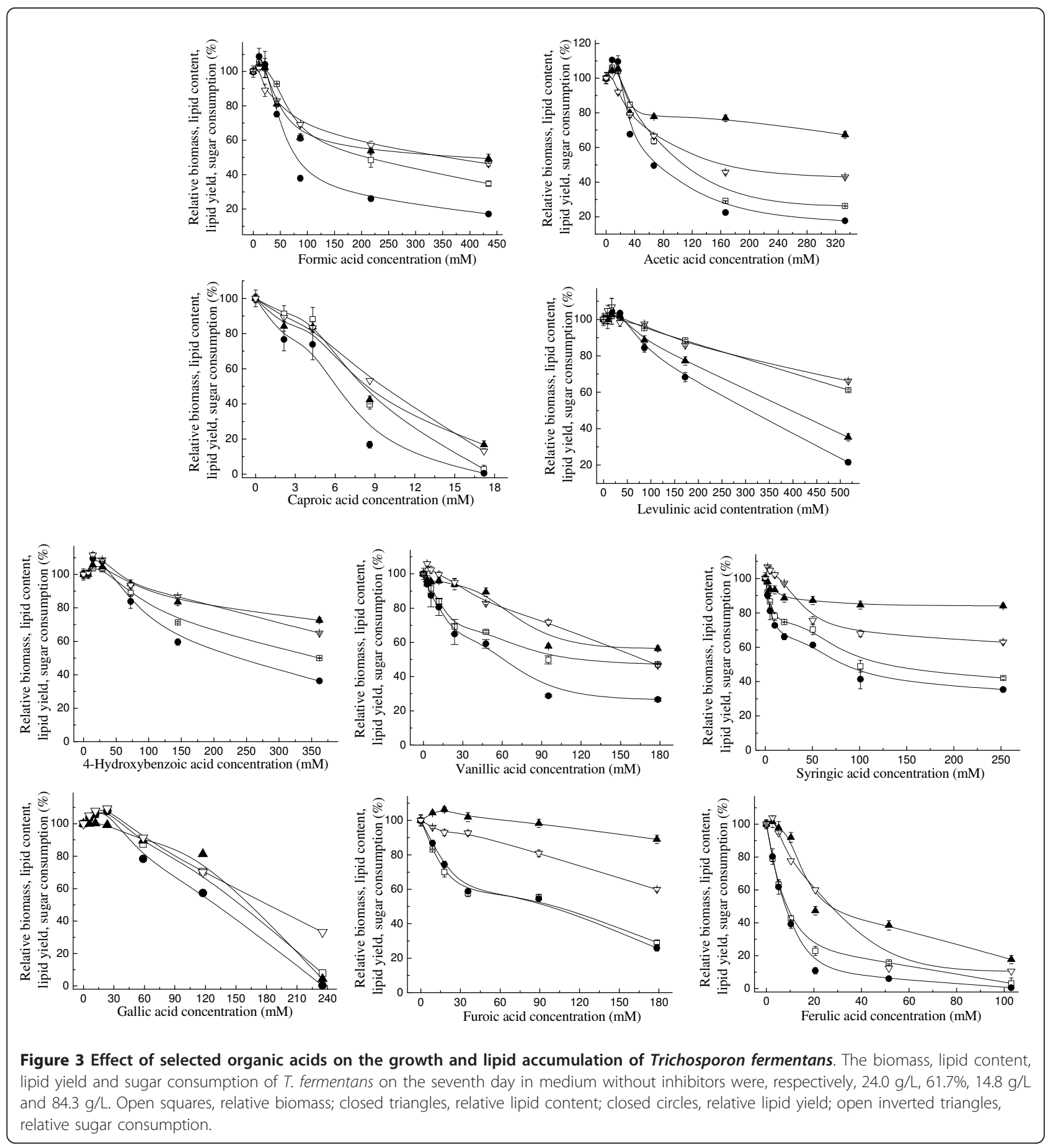

supplemented with Teucrium polium extract (in quantities of $10 \mathrm{~g} / \mathrm{L}$ ) [22]. More recently, Y. lipolytica has been further proven to produce substantially higher SCO quantities in media supplemented with phenol compounds (concentrations ranging from 5 to $10 \mathrm{mM}$ ) [23].

$\mathrm{IC}_{25}$ and $\mathrm{IC}_{50}$, which represent the molar concentrations of the tested organic acids that cause $25 \%$ and 50\% inhibition of the lipid yield of $T$. fermentans, respectively, are summarized in Table 2 . The relative biomass and lipid content at $\mathrm{IC}_{25}$ and $\mathrm{IC}_{50}$ concentrations are also given in Table 2. Apparently, there is no direct relationship between the toxicity and hydrophobicity of organic acid, which does not agree with the previous report describing ethnologenic Escherichia coli, in which the investigators found that the more hydrophobic the organic acid, the stronger its inhibitory effect 
Table 2 Concentration of organic acids required to inhibit the lipid yield of Trichosporon fermentans

\begin{tabular}{|c|c|c|c|c|c|c|c|}
\hline Organic acid & $\begin{array}{l}\mathrm{IC}_{25}^{\mathrm{a}} \\
(\mathrm{mM})\end{array}$ & $\begin{array}{c}\text { Relative biomass } \\
\text { (\%) }\end{array}$ & $\begin{array}{c}\text { Relative lipid } \\
\text { content (\%) }\end{array}$ & $\begin{array}{l}\mathrm{IC}_{50}^{\mathrm{b}} \\
(\mathrm{mM})\end{array}$ & $\begin{array}{c}\text { Relative biomass } \\
\text { (\%) }\end{array}$ & $\begin{array}{l}\text { Relative lipid } \\
\text { content (\%) }\end{array}$ & $\log P^{c}$ \\
\hline Formic acid & 43.4 & 92 & 81 & 80.4 & 72 & 68 & -0.54 \\
\hline Acetic acid & 30.0 & 87 & 83 & 66.6 & 68 & 78 & -0.17 \\
\hline Levulinic acid & 146.4 & 90 & 82 & 310.0 & 79 & 61 & -0.49 \\
\hline 4-hydroxybenzoic acid & 97.7 & 84 & 89 & 188.2 & 67 & 80 & 1.58 \\
\hline Vanillic acid & 15.5 & 80 & 93 & 60.7 & 63 & 79 & 1.43 \\
\hline Syringic acid & 9.6 & 79 & 94 & 78.2 & 58 & 86 & 1.04 \\
\hline Furoic acid & 17.8 & 70 & 106 & 93.7 & 52 & 98 & 1.06 \\
\hline Gallic acid & 67.6 & 88 & 86 & 135.2 & 63 & 74 & 0.7 \\
\hline Ferulic acid & 3.1 & 76 & 99 & 7.7 & 51 & 97 & 1.51 \\
\hline Caproic acid & 3.0 & 88 & 83 & 5.6 & 71 & 69 & 1.92 \\
\hline
\end{tabular}
[24].

[24]. In most cases, the molar concentrations of $\mathrm{IC}_{25}$ and $\mathrm{IC}_{50}$ for organic acids are higher than the corresponding values for aldehydes [25]. For example, the $\mathrm{IC}_{50}$ of vanillic acid was $60.7 \mathrm{mM}$ but $6.6 \mathrm{mM}$ for vanillin, suggesting that organic acids are less toxic than aldehydes to lipid production of $T$. fermentans.

The sugar consumption in the medium containing the selected organic acid was also recorded after fermentation for seven days at which the control without inhibitor gave the maximum lipid yield and the residual sugar was xylose with a concentration of about $15.7 \mathrm{~g} / \mathrm{L}$ (Figure 3). Interestingly, except for furoic and caproic acids, the relative sugar consumption was above $100 \%$ in the presence of a small amount of organic acids. However, improved sugar utilization did not necessarily lead to an enhanced lipid yield. For example, T. fermentans could utilize more sugars than the control in the presence of 5 $\mathrm{mM}$ syringic acid, but the corresponding relative lipid yield was only $81.1 \%$. At higher concentrations, however, all the tested organic acids suppressed the sugar utilization, and the higher the concentration, the more pronounced the suppression. Among the organic acids examined, levulinic acid showed the least influence on sugar utilization, which is in accordance with the observation that levulinic acid displayed the lowest toxicity to lipid production of $T$. fermentans.

To gain deeper insight into the sugar assimilation, the time course of sugar utilization in the medium containing the selected organic acid at its $\mathrm{IC}_{25}$ was further investigated. As shown in Figures 4A and 4D, the glucose consumption rate decreased in the presence of acids, but it still could be exhausted by $T$. fermentans with an increase in time, as reported by Narendranath et al. [26] and Huang et al. [12]. Similarly, aliphatic acids decreased the xylose consumption rate throughout the fermentation process, and less xylose was utilized compared with the control (Figure 4B). In the presence of aromatic or furan acids such as vanillic, syringic, furoic and ferulic acids, however, T. fermentans can utilize xylose at a faster rate than the control from day 5 , and more xylose was consumed at the end of fermentation (Figure 4E). Obviously, in some cases, organic acids can stimulate the utilization of xylose. However, the enhanced sugar conversion did not result in improved lipid production. A similar phenomenon was also observed in our previous work on the effect of aldehyde on the growth and lipid accumulation of $T$. fermentans [25]. The reason for this is now under investigation in our laboratory.

In our previous studies $[10,12]$, we found that when glucose is almost exhausted, cellular lipids can be used as a carbon source to maintain the growth of $T$. fermentans. In general, microorganisms consume their accumulated lipids mainly through the glyoxylate bypass pathway, and, more specifically, different microbes might preferentially consume different kinds of fatty acids to maintain their growth [6]. In this work, there is also apparent cellular lipid degradation between the seventh and tenth days for control without inhibitors. A similar tendency was observed in the culture of $T$. fermentans on the media containing various organic acids (data not shown). The lipid degradation rate is slower than the control, however, because of the presence of acids, especially the aromatic acids, suggesting that organic acids repress lipid turnover as well. This is an interesting phenomenon because repression of accumulated lipid degradation has been observed only in multiple limited media [27].

Malic enzyme is considered the key enzyme for lipid synthesis in oleaginous microorganisms because it is one of the main enzymes providing a supply of NADPH (nicotinamide adenine dinucleotide phosphate oxidase) to microorganisms $[28,29]$. In this work, the effects of the selected organic acids (each at its $\mathrm{IC}_{25}$ concentration) on the malic enzyme activity were tested after the second day of fermentation, when the lipid formation 

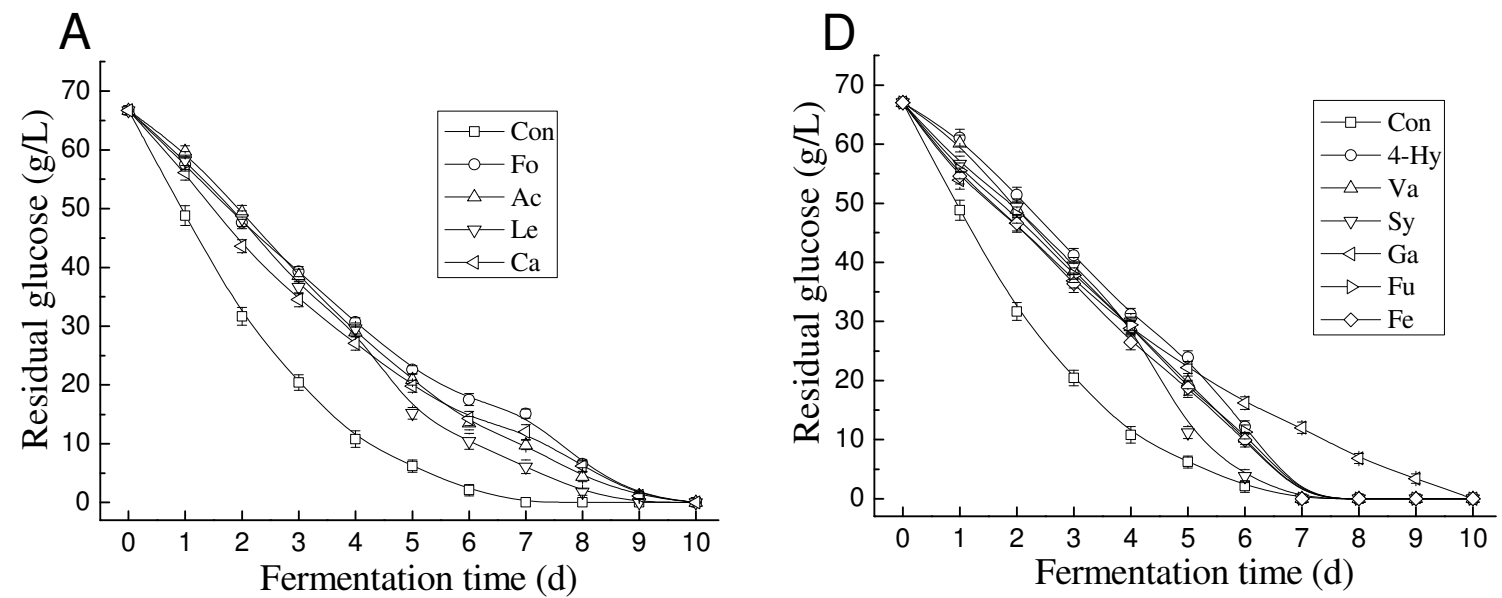

$\mathrm{B}$

E
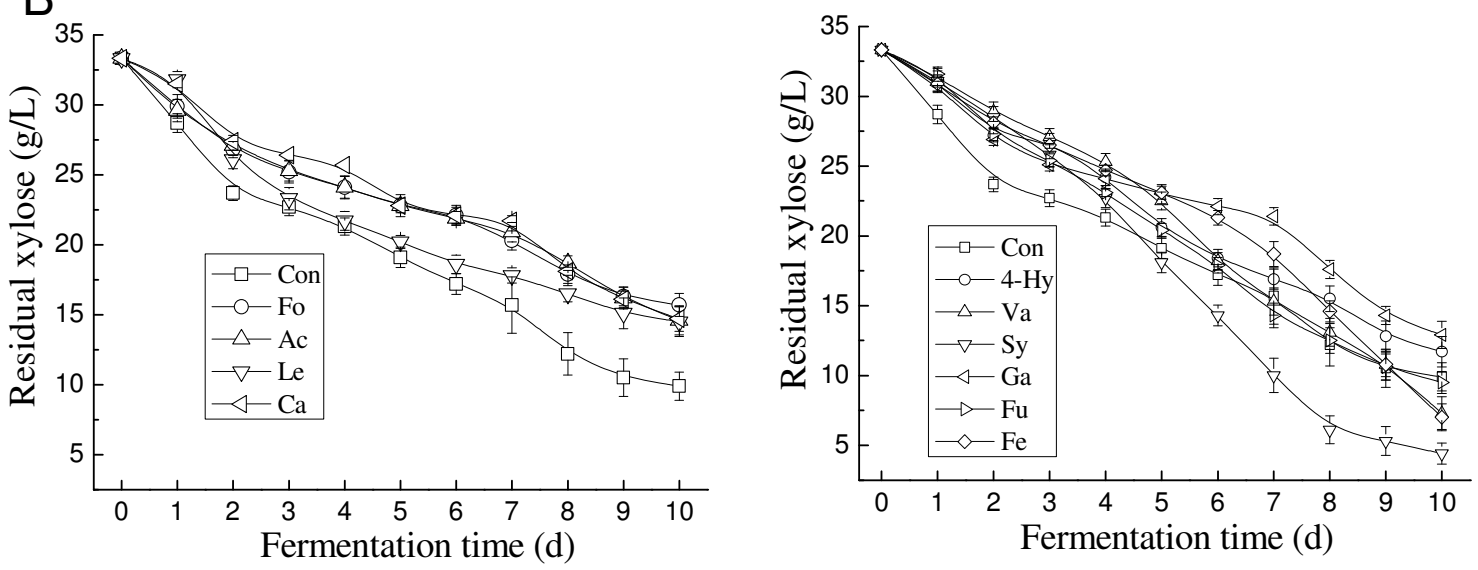

C
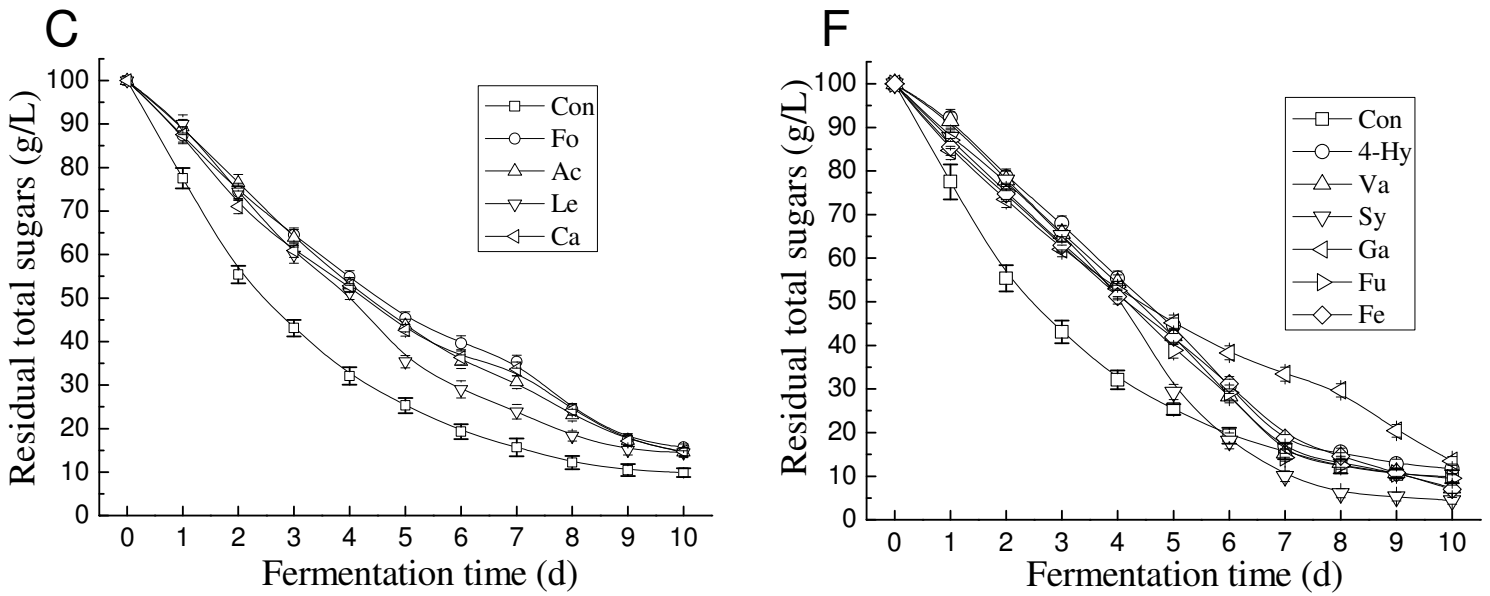

Figure 4 Effect of the selected organic acids on the sugar metabolism of Trichosporon fermentans. (A) and (D) Glucose consumption. (B) and (E) Xylose consumption. (C) and (F) Total sugar consumption. Ac, acetic acid; Fo, formic acid; Le, levulinic acid; Hy, 4-hydroxybenzoic acid; Fu, furoic acid; Ca, caproic acid; Ga, gallic acid; Fe, ferulic acid; Sy, syringic acid; Va, vanillic acid. 


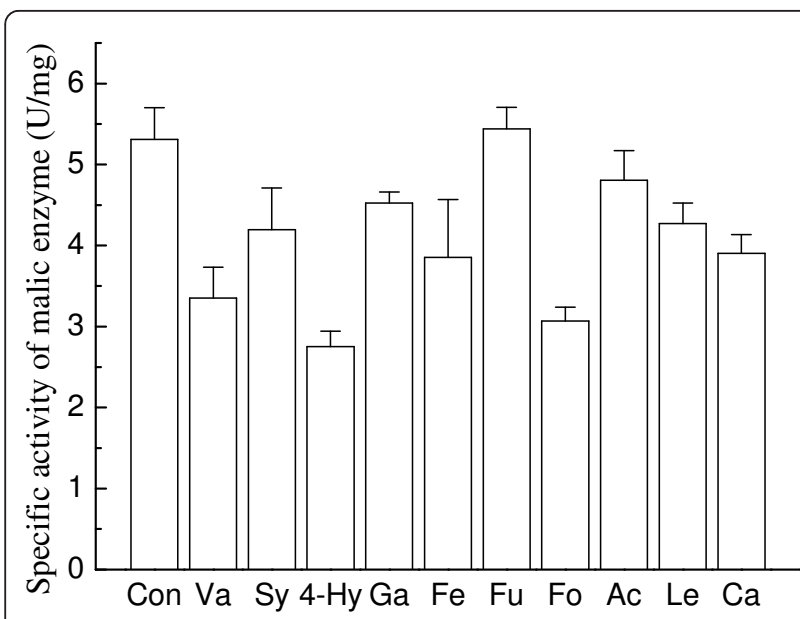

Figure 5 Effects of selected organic acids on malic enzyme activity in Trichosporon fermentans. Con, control; Ac, acetic acid; Fo, formic acid; Le, levulinic acid; Hy, 4-hydroxybenzoic acid; Fu, furoic acid; Ca, caproic acid; Ga, gallic acid; Fe, ferulic acid; Sy, syringic acid; Va, vanillic acid.

rate reached the maximum in culture without an inhibitor. As shown in Figure 5, the malic enzyme activity was inhibited by all the organic acids tested except furoic acid, which can well explain the delay in lipid accumulation in the media containing these acids. Interestingly, for furoic acid, there was no significant change in the specific activity of malic enzyme, and even a higher malic enzyme activity was detected, which partly explains the small influence of furoic acid on the lipid synthesis of T. fermentans mentioned above (Figure 3).

In many cases, addition of natural compounds, such as sterculia oil $[4,5]$, essential oils [30], plant extracts [31] or phenol-type compounds [20], even in small concentrations, can have profound effects upon the fatty acid composition of microbial lipids. High-addedvalue microbial lipids such as cocoa butter substitutes can be produced by modifying the fatty acid composition of lipids under various conditions $[4,5]$. The effect of organic acids on the fatty acid composition of lipids from T. fermentans is shown in Table 3. Apparently, oleic acid was the most abundant fatty acid, being about $50 \%$ to $60 \%$ of the total fatty acids, followed by palmitic acid, stearic acid and linoleic acid. In most cases, organic acid had no significant influence on the composition of unsaturated acids, including oleic acid and linoleic acid. However, the presence of organic acid would increase the palmitic acid content. The stearic acid content varied with the organic acid. It is worth noting that acetic acid, the most abundant acid in the lignocellulosic hydrolysates, had little influence on the fatty acid composition of lipid produced by $T$. fermentans.
Effects of inoculum size, temperature, and initial $\mathrm{pH}$ on the inhibition by organic acids

It has been reported that the toxicity of organic acids to microorganism can be relieved by increasing cell density in fermentation [24]. Therefore, each selected organic acid was added to the medium at its $\mathrm{IC}_{50}$ concentration to examine the effect of inoculum size on the inhibition of the organic acid (Figure 6). In most cases, increasing inoculum size cannot reduce the inhibitory effects of acids on cell growth and lipid accumulation. Unexpectedly, in the case of caproic acid, high inoculum size (10\% and $15 \%$ ) not only eliminated its inhibition on cell growth but also enhanced cell growth, as indicated by a relative biomass of more than $100 \%$.

The effects of temperature and initial $\mathrm{pH}$ on the inhibition by organic acids at their respective $\mathrm{IC}_{50}$ concentrations were also recorded. No direct correlation between the culture temperature and the inhibitory effect of acids was observed (Figure 7). Similarly, there was no relationship between the initial $\mathrm{pH}$ and the inhibition by acids (Figure 8). Initial pH, however, exerted a greater impact than temperature on inhibition. Within the range of $\mathrm{pH}$ levels tested, for most of aliphatic acids, a higher initial $\mathrm{pH}$ resulted in a lower relative biomass. Interestingly, an opposite result was obtained in the presence of most aromatic or furanacids. It is worth noting that in some cases, a suitable $\mathrm{pH}$ can remarkably relieve and even eliminate the inhibitory effect of acids. For example, in the presence of gallic acid, the biomass and lipid content were only 0.8 $\mathrm{g} / \mathrm{L}$ and $12.6 \%$, respectively, at $\mathrm{pH} 7.5$ (with corresponding relative biomass and lipid content being 3.8\% and 22.4\%). However, the toxicity of gallic acid dropped sharply with the decrease of $\mathrm{pH}$ and even an enhanced effect on cell growth and lipid accumulation was observed at pH 5.5, at which the biomass and lipid content reached as high as $23.1 \mathrm{~g} / \mathrm{L}$ and $59.9 \%$, respectively (with the relative biomass and lipid content being $125.3 \%$ and $104.8 \%$ ).

\section{Effects of binary combinations of organic acids on cell growth and lipid accumulation of $T$. fermentans}

It has been reported that the synergistic effect of different inhibitors present in the lignocellulosic hydrolysates is complex [32-34]. Therefore, the effects of binary combinations of organic acids on the cell growth and lipid accumulation of $T$. fermentans were tested at their respective $\mathrm{IC}_{25}$ concentrations listed in Table 2. Acetic and 4-hydroxybenzoic acids, the typical aliphatic and aromatic acids in lignocellulosic hydrolysate, respectively, were chosen for binary combinations with other organic acids. In the experiments, whenever two acids were combined, the predicted relative biomass, lipid content and lipid yield represented the values after deduction of the summed inhibition on biomass, lipid content and lipid yield by each of the two tested 
Table 3 Effect of organic acids on the fatty acid composition of lipids

\begin{tabular}{|c|c|c|c|c|c|}
\hline \multirow[b]{2}{*}{ Organic acid } & \multicolumn{5}{|c|}{ Fatty acid composition (\%) } \\
\hline & Palmitic acid (C16:0) & Linoleic acid (C18:2) & Oleic acid (C18:1) & Stearic acid (C18:0) & Others \\
\hline Acetic acid (2 g/L) & 19.8 & 6.6 & 55.9 & 16.5 & 1.2 \\
\hline Syringic acid (0.5 g/L) & 22.9 & 5.9 & 47.6 & 22.6 & 1 \\
\hline Vanillic acid (0.5 g/L) & 23.6 & 5.8 & 47.1 & 22.5 & 1 \\
\hline Caproic acid (0.25 g/L) & 24 & 6.8 & 56.9 & 10.9 & 1.4 \\
\hline Furoic acid $(0.5 \mathrm{~g} / \mathrm{L})$ & 26.1 & 5.6 & 53 & 14.4 & 0.9 \\
\hline 4-hydroxybenzoic acid (0.5 g/L) & 21.2 & 5.8 & 54.1 & 17.9 & 1 \\
\hline Formic acid (1 g/L) & 19.5 & 6.6 & 57.8 & 15.2 & 0.9 \\
\hline Levulinic acid (4 g/L) & 22.6 & 5.6 & 54.2 & 15.8 & 1.8 \\
\hline Ferulic acid (2 g/L) & 20.7 & 4.8 & 60.4 & 11.8 & 2.3 \\
\hline Gallic acid (4 g/L) & 17.2 & 7.3 & 56.6 & 18.3 & 0.6 \\
\hline Control & 17.4 & 6.1 & 57.9 & 17.4 & 1.2 \\
\hline
\end{tabular}

avalues in parentheses represent the organic acid's concentration tested.

inhibitors at their $\mathrm{IC}_{25}$ concentrations. If the actual experimental value exceeded the predicted value, the inhibition was referred to as "synergistic."

As shown in Figure 9A, taking lipid yield into account for the binary combinations of acetic acid with other organic acids, the inhibition caused by most of the binary combinations was roughly equal to the predicted value. However, the combination of acetic acid with formic or gallic acid showed a synergistic inhibition on lipid yield. For example, the combination of acetic acid and gallic acid led to a $95 \%$ decrease in lipid yield compared to the predicted value. Likewise, in the cases of binary combinations of 4-hydroxybenzoic acid with other organic acids (Figure 9B), most of the binary combinations caused no synergistic inhibition. Interestingly, the binary combination of 4-hydroxybenzoic acid and caproic acid greatly reduced the inhibition on cell growth and lipid accumulation and resulted in a $60 \%$ increase in the lipid yield compared to the predicted value.

\section{Conclusions}

The inhibitory effect of organic acids on the cell growth and lipid accumulation of T. fermentans can be relieved or even eliminated at low concentrations. Thus, more efforts should be made to improve the stress assistance of T. fermentans to inhibitors by genetic engineering and/or domestic methods. The development of cost-effective hydrolytic or detoxification processes to obtain lignocellulosic hydrolysates with lower inhibitor concentrations would also be useful. The inhibition of organic acids can also be reduced by optimizing culture conditions, such as inoculum size, temperature and initial $\mathrm{pH}$ of the medium.

\section{Methods}

\section{Microorganism and chemicals}

Oleaginous yeast T. fermentans CICC 1368 was obtained from the China Center of Industrial Culture Collection and kept on wort agar at $4^{\circ} \mathrm{C}$. Levulinic acid, 4- hydroxybenzoic acid, syringic acid, vanillic acid and furoic acid were purchased from Alfa Aesar (Heysham, UK). Acetic acid, formic acid, caproic acid, ferulic acid, gallic acid and other chemicals were obtained from commercial sources and were of the highest purity available.

\section{Medium, precultivation and cultivation}

The composition of the precultivation medium ( $\mathrm{pH}$ 6.0) was as follows: glucose and xylose $20 \mathrm{~g} / \mathrm{L}$ (ratio 2:1 wt/wt), $10 \mathrm{~g} / \mathrm{L}$ peptone and $10 \mathrm{~g} / \mathrm{L}$ yeast extract. The composition of the fermentation medium (pH 6.5) was as follows: $100 \mathrm{~g} /$ $\mathrm{L}$ glucose and xylose (ratio $2: 1 \mathrm{wt} / \mathrm{wt}$ ), $1.8 \mathrm{~g} / \mathrm{L}$ peptone, 0.5 $\mathrm{g} / \mathrm{L}$ yeast extract, $0.4 \mathrm{~g} / \mathrm{L} \mathrm{MgSO}_{4} \cdot 7 \mathrm{H}_{2} \mathrm{O}, 2.0 \mathrm{~g} / \mathrm{L} \mathrm{KH}_{2} \mathrm{PO}_{4}$, $0.003 \mathrm{~g} / \mathrm{L} \mathrm{MnSO} \mathrm{M}_{4} \cdot \mathrm{H}_{2} \mathrm{O}$ and $0.0001 \mathrm{~g} / \mathrm{L} \mathrm{CuSO}_{4} \cdot 5 \mathrm{H}_{2} \mathrm{O}$.

The preculture was performed in a $250-\mathrm{ml}$ conical flask containing $50 \mathrm{ml}$ of precultivation medium in a rotary shaker at $28^{\circ} \mathrm{C}$ and $160 \mathrm{rpm}$ for 24 hours. Next, $5 \%$ seed culture $(2.5 \mathrm{ml})$ was inoculated in a $250-\mathrm{ml}$ conical flask containing $47.5 \mathrm{ml}$ of fermentation medium, and cultivation was carried out in a rotary shaker at $25^{\circ} \mathrm{C}$ and $160 \mathrm{rpm}$ for seven days.

\section{Effect of sugar concentration on the growth and lipid accumulation of $T$. fermentans}

A mixture of glucose and xylose at a ratio of 2:1 (wt/wt) was used as the carbon source. The medium, at an initial sugar concentration of $25,50,75,100,125,150$, 200,300 or $400 \mathrm{~g} / \mathrm{L}$, respectively, was used for the substrate inhibition study. After five days' fermentation, the biomass, lipid content, lipid yield and sugar concentration of $T$. fermentans on the medium with different initial sugar concentrations were compared.

\section{Effects of organic acids on the growth and lipid accumulation}

After precultivation, $2.5 \mathrm{ml}$ of seed culture were inoculated in $47.5 \mathrm{ml}$ of fermentation medium containing the selected 


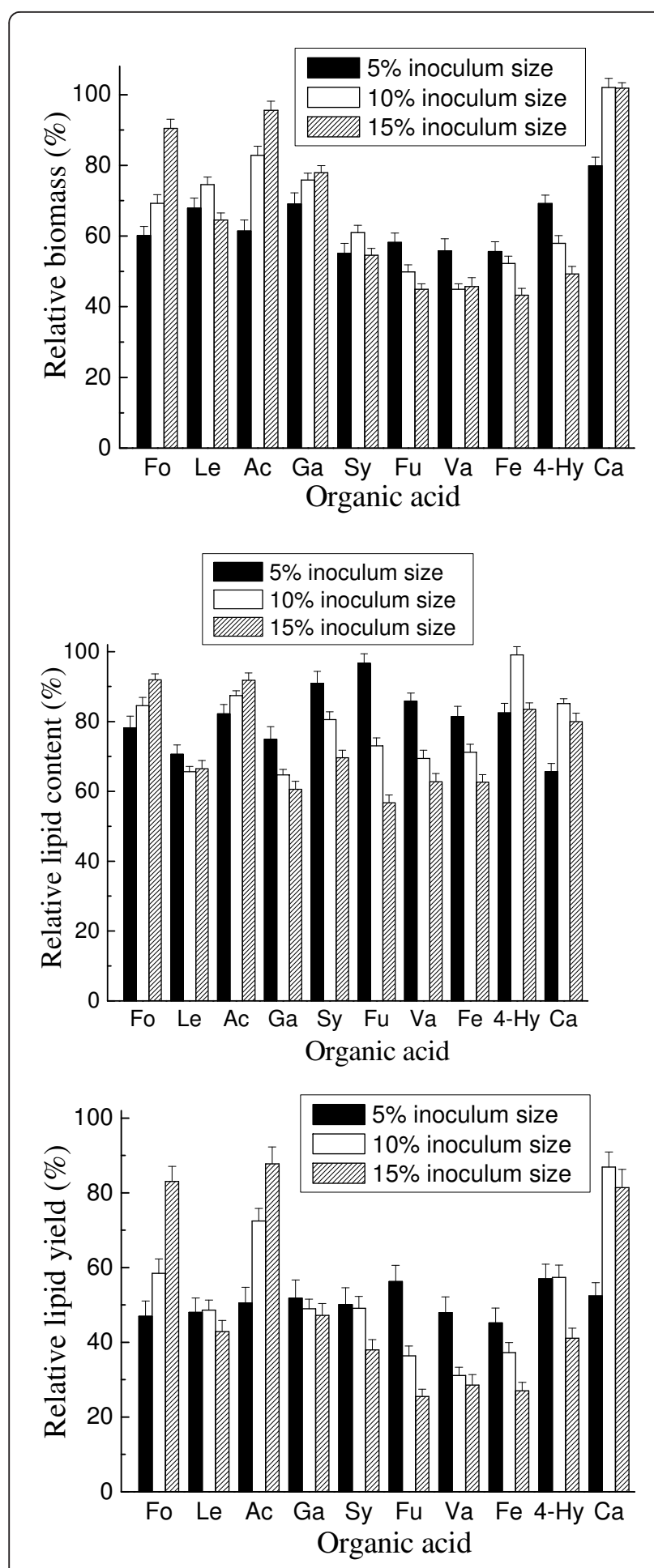

Figure 6 Effect of inoculum size on the inhibition of organic acids. Each organic acid was tested at the molar concentration of the tested organic acids that caused 50\% inhibition of the lipid yield of Trichosporon fermentans. Cultures were incubated initially at $\mathrm{pH} 6.5,25^{\circ} \mathrm{C}$ and $160 \mathrm{rpm}$ for 7 days. The results are expressed relative to controls without organic acids. Biomass, lipid content and lipid yield of cultures in the absence of organic acids with 5\%, 10\% and $15 \%$ inoculum sizes were, respectively, $24.0 \mathrm{~g} / \mathrm{L}, 61.7 \%$ and 14.8 $\mathrm{g} / \mathrm{L} ; 22.4 \mathrm{~g} / \mathrm{L}, 58.6 \%$ and $13.1 \mathrm{~g} / \mathrm{L} ;$ and $21.6 \mathrm{~g} / \mathrm{L}, 54.3 \%$ and $11.7 \mathrm{~g} / \mathrm{L}$. Ac, acetic acid; Fo, formic acid; Le, levulinic acid; $\mathrm{Hy}$, 4hydroxybenzoic acid; Fu, furoic acid; $\mathrm{Ca}$, caproic acid; Ga, gallic acid; Fe, ferulic acid; Sy, syringic acid; Va, vanillic acid.
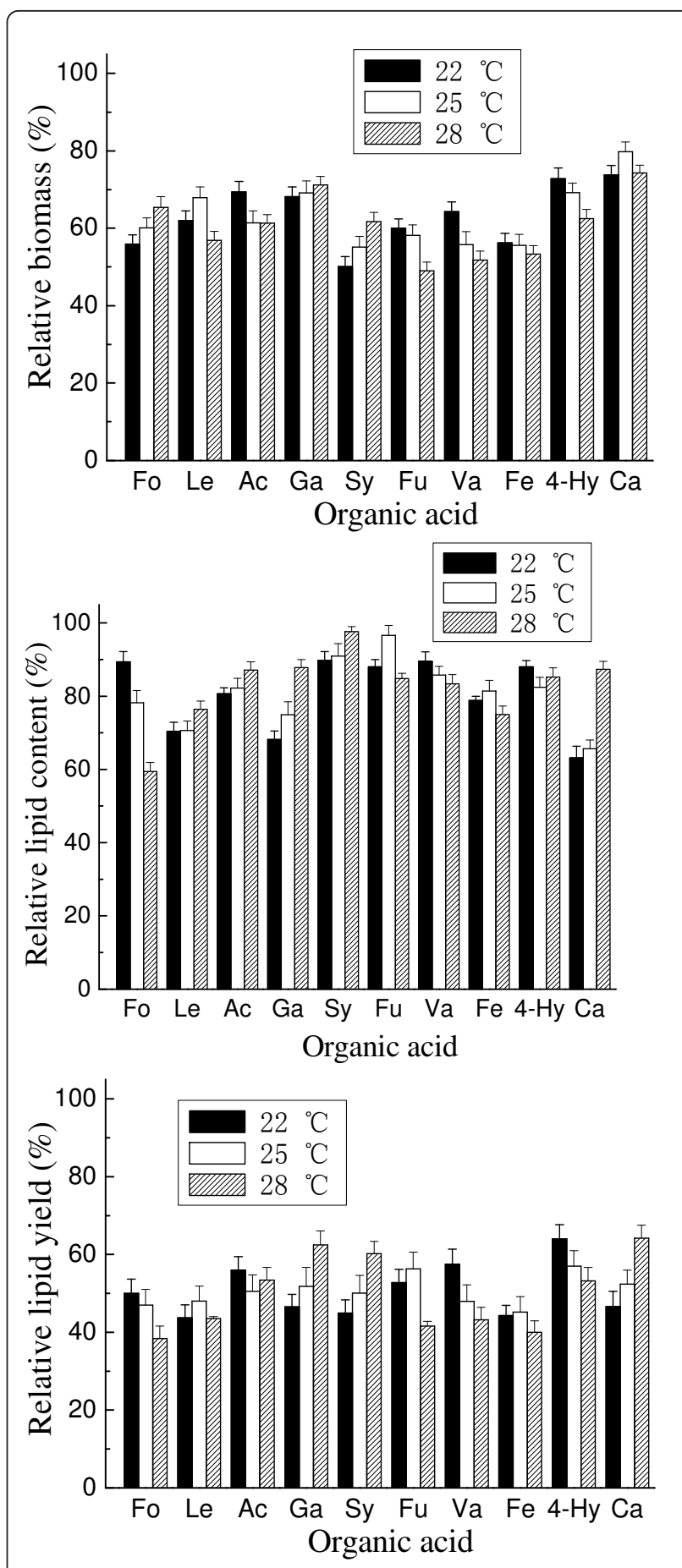

Figure 7 Effect of temperature on the inhibition of organic acids. All organic acids were tested at their respective molar concentrations of the tested organic acids that caused 50\% inhibition of the lipid yield. Cultures with $5 \%$ inoculum size were incubated at initial pH 6.5 and 160 rpm for 7 days. Results are expressed relative to controls without organic acids. Biomass, lipid content and lipid yield of cultures lacking organic acids at $22^{\circ} \mathrm{C}, 25^{\circ}$ $\mathrm{C}$ and $28^{\circ} \mathrm{C}$ were, respectively, $19.9 \mathrm{~g} / \mathrm{L}, 55.8 \%$ and $11.1 \mathrm{~g} / \mathrm{L} ; 24.0 \mathrm{~g} /$ $\mathrm{L}, 61.7 \%$ and $14.8 \mathrm{~g} / \mathrm{L} ;$ and $23.6 \mathrm{~g} / \mathrm{L}, 58.9 \%$ and $13.9 \mathrm{~g} / \mathrm{L}$. Ac, acetic acid; Fo, formic acid; Le, levulinic acid; Hy, 4-hydroxybenzoic acid; Fu, furoic acid; Ca, caproic acid; Ga, gallic acid; Fe, ferulic acid; Sy, syringic acid; $V a$, vanillic acid. 

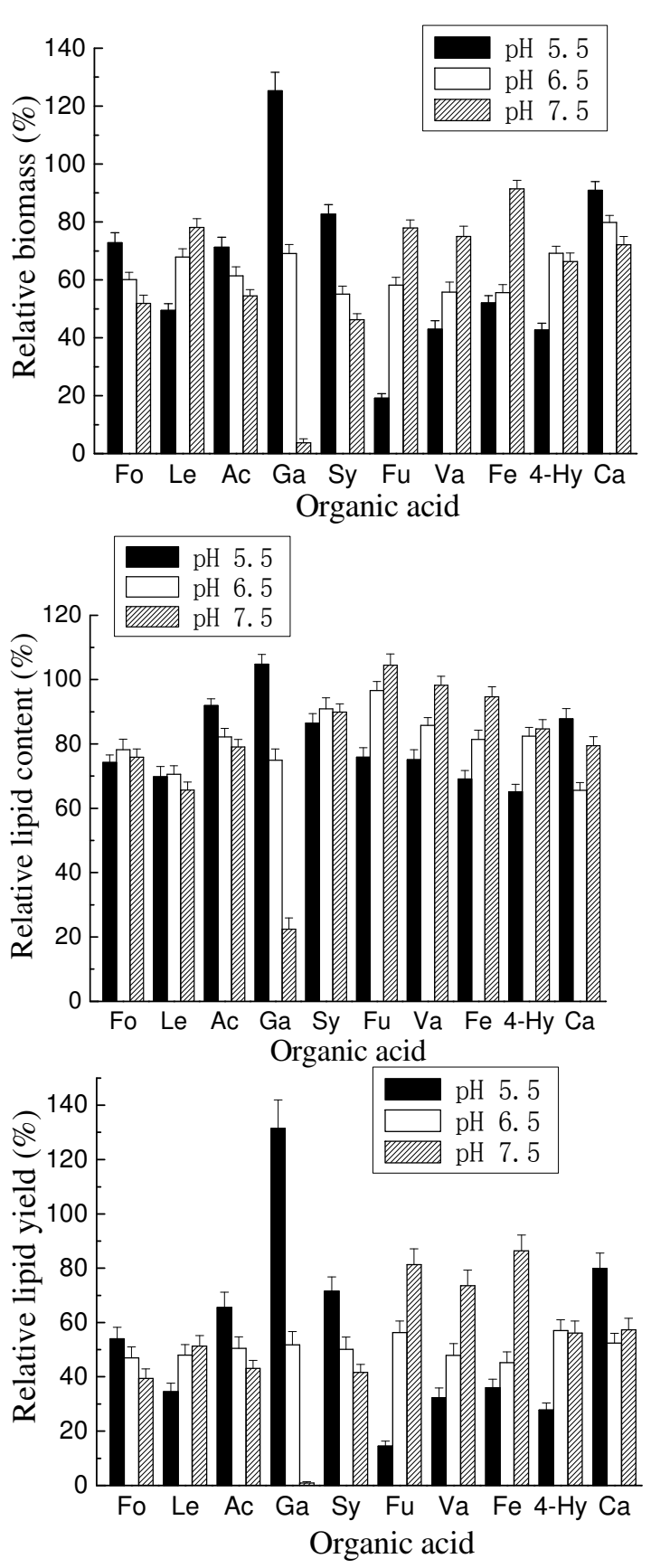

Figure 8 Effect of initial pH on the inhibition of organic acids All organic acids were tested at their respective molar concentrations of the tested organic acids that caused 50\% inhibition of the lipid yield. Cultures with $5 \%$ inoculum size were incubated at $25^{\circ} \mathrm{C}$ and $160 \mathrm{rpm}$ for 7 days. Results are expressed relative to controls without organic acids. Biomass, lipid content and lipid yield of cultures lacking organic acids at $\mathrm{pH} 5.5, \mathrm{pH} 6.5$ and $\mathrm{pH}$ 7.5 were, respectively, $18.4 \mathrm{~g} / \mathrm{L}, 57.2 \%$ and $10.5 \mathrm{~g} / \mathrm{L} ; 24.0 \mathrm{~g} / \mathrm{L}, 61.7 \%$ and $14.8 \mathrm{~g} / \mathrm{L}$; and $21.5 \mathrm{~g} / \mathrm{L}, 56.3 \%$ and $12.1 \mathrm{~g} / \mathrm{L}$. Ac, acetic acid; Fo, formic acid; Le, levulinic acid; Hy, 4-hydroxybenzoic acid; Fu, furoic acid; Ca, caproic acid; Ga, gallic acid; Fe, ferulic acid; Sy, syringic acid; Va, vanillic acid. organic acid. To facilitate averaging, results throughout the text are expressed as percentages of the control values without addition of the tested inhibitor (with the biomass, lipid content, lipid yield and residual sugar concentration after seven days' fermentation being $24.0 \mathrm{~g} / \mathrm{L}, 61.7 \%, 14.8$ $\mathrm{g} / \mathrm{L}$ and $15.7 \mathrm{~g} / \mathrm{L}$, respectively). $\mathrm{IC}_{25}$ and $\mathrm{IC}_{50}$, defined as the molar concentrations of the tested organic acids that cause $25 \%$ and $50 \%$ inhibition of the lipid yield of T. fermentans, respectively, were measured according to the data shown in Figure 3. All reported data were averages of experiments performed at least in triplicate.

\section{Effects of inoculum size, temperature and initial $\mathrm{pH}$ on the inhibition of organic acids}

The effects of inoculum size, temperature or initial $\mathrm{pH}$ on the potency of organic acids were examined using acid concentrations of $\mathrm{IC}_{50}$. For inoculum size, 5\%, 10\% and $15 \%$ seed culture were inoculated in the fermentation media containing the selected acids $\left(\mathrm{IC}_{50}\right)$. For temperature, the cultures with $5 \%$ inoculum size were maintained at $22^{\circ} \mathrm{C}, 25^{\circ} \mathrm{C}$ and $28^{\circ} \mathrm{C}$, respectively. Fermentation media containing the assayed acids were adjusted to $\mathrm{pH} 5.5,6.5$ or 7.5 prior to inoculation to test the effect of initial $\mathrm{pH}$. Biomass, lipid content and lipid yield were all measured after seven days' fermentation.

\section{Binary combinations of organic acids}

Two selected organic acids with each concentration at $\mathrm{IC}_{25}$ were added to the fermentation medium. Cultures were inoculated as described above and incubated for seven days $\left(5 \%\right.$ inoculum size, $\mathrm{pH} 6.5$, and $\left.25^{\circ} \mathrm{C}\right)$. Cultures grown without adding organic acids were used as the control.

\section{Effects of organic acids on sugar utilization and malic enzyme activity}

The effects of organic acids on sugar utilization were examined with the acid concentration being $\mathrm{IC}_{25}$. The relative sugar consumption was defined as the ratio of the amount of glucose and xylose consumed by the yeast cells grown on the culture medium containing the selected organic acid for seven days to that without the acid. The malic enzyme activity of T. fermentans was measured according to our previous work [25] with a SHIMADZU UV-2550 spectrophotometer (Kyoto, Japan).

\section{Analytical methods}

Biomass was harvested by centrifugation and weighed in its lyophilized form [35]. Extraction of lipid from lyophilized biomass was performed according to a procedure modified from the one described by Folch et al. [36], with a mixture of chloroform and methanol $(2: 1 \mathrm{vol} /$ vol). The extracted lipid was centrifuged to obtain a 

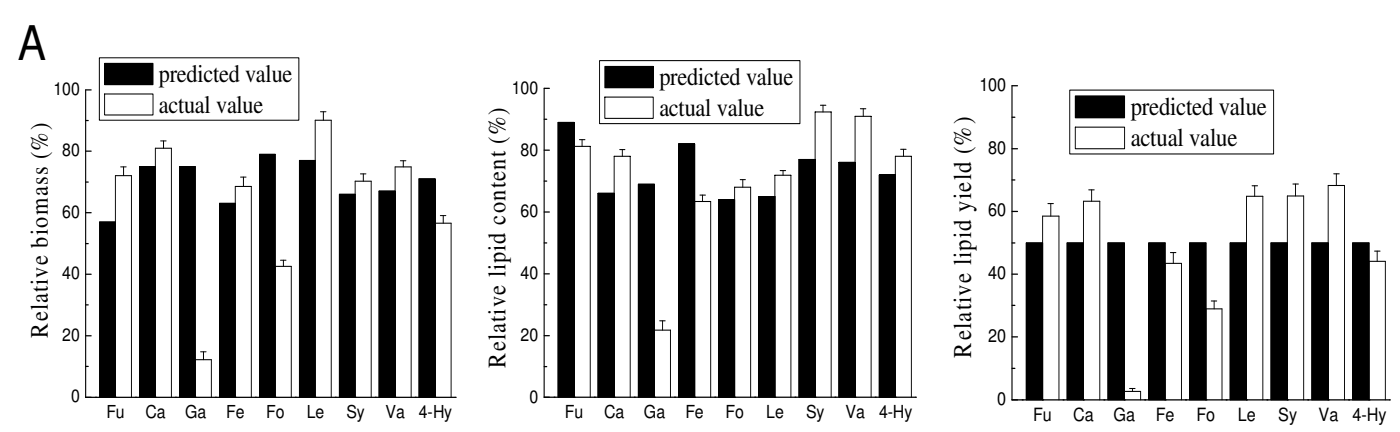

Binary combination with acetic acid $\left(\mathrm{IC}_{25}\right)$
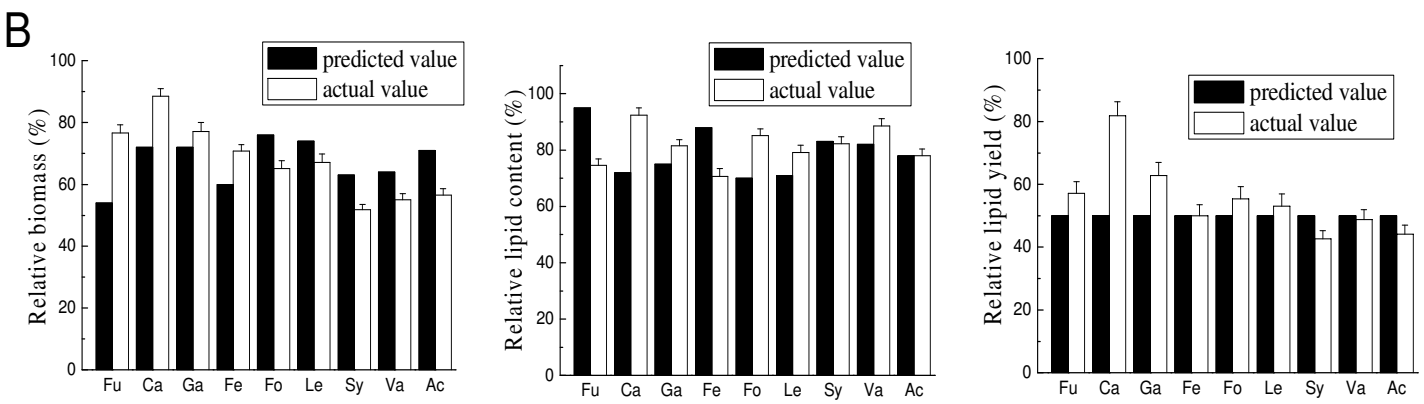

Binary combination with 4-hydroxybenzoic acid $\left(\mathrm{IC}_{25}\right)$

Figure 9 Effect of binary combinations on the growth and lipid accumulation of Trichosporon fermentans. (A) Acetic acids (molar concentration of the tested organic acids that caused 25\% inhibition of the lipid yield (IC $\left.C_{25}\right)$ ). (B) 4-hydroxybenzoic acid (IC 25$)$. AC, acetic acid; Fo, formic acid; Le, levulinic acid; Hy, 4-hydroxybenzoic acid; Fu, furoic acid; Ca, caproic acid; Ga, gallic acid; Fe, ferulic acid; Sy, syringic acid; Va, vanillic acid.

clear supernatant, and the solvent was removed by evaporation under a vacuum at $100 \mathrm{hPa}, 55^{\circ} \mathrm{C}$ and $100 \mathrm{rpm}$ (EYELA NE series rotary evaporator; Tokyo Rikakikai Co, Ltd, Tokyo, Japan). Lipid yield is expressed as the amount of lipid extracted from the cells in per liter of fermentation broth $(\mathrm{g} / \mathrm{L})$, and lipid content is defined as the percentage of lipid to dry biomass (\% wt/wt). The fatty acid profile of the lipid from T. fermentans was determined by gas chromatography (GC-2010 Plus; Tokyo Rikakikai Co, Ltd) with an ionization detector and a DB- 1 capillary column $(0.25 \mathrm{~cm} \times 30 \mathrm{~m}$; Agilent Technologies Inc, Santa Clara, CA, USA) according to previously published procedures [10]. D-xylose and Dglucose were measured by high-performance liquid chromatography (Waters Corp, Milford, MA, USA) with a differential refractive index detector (Waters 2410; Waters Corp) and an Aminex HPX-87P column (300 $\mathrm{mm} \times 7.8 \mathrm{~mm}$; Bio-Rad Laboratories, Hercules, CA, USA) at $85^{\circ} \mathrm{C}$. Deionized water was used as the mobile phase at $0.5 \mathrm{~mL} / \mathrm{minute}$.

\section{Abbreviations}

$I_{25}$ and $I C_{50}$ : the molar concentrations of the tested organic acids that causes $25 \%$ and $50 \%$ inhibition on the lipid yield of T. fermentans; Con: Control trial on the medium without inhibitor; Ac: acetic acid; Fo: formic acid; Le: levulinic acid; Hy: 4-hydroxybenzoic acid; Fu: furoic acid; Ca: caproic acid; Ga: gallic acid; Fe: ferulic acid; Sy: syringic acid; Va: vanillic acid.

\section{Acknowledgements}

We acknowledge the Major State Basic Research Development Program "973" (grant 2010CB732201), the National Natural Science Foundation of China (grants 31071559, 21072065 and 20876059), the Science and Technology Project of Guangdong Province (grants 2009B080701085 and 2009B030801004), the Doctoral Program of Higher Education (grant 20090172110019), the Open Project Program of the State Key Laboratory of Pulp and Paper Engineering, SCUT (grant 201138), and the Open Project Program for Key Laboratory of Fermentation Engineering (Ministry of Education), Hubei University of Technology for financial support.

\section{Author details}

${ }^{1}$ State Key Laboratory of Pulp and Paper Engineering, College of Light Industry and Food Sciences, South China University of Technology, 381 Wushan Rd.,Tianhe District, Guangzhou 510640, China. ${ }^{2}$ School of Biosciences and Bioengineering, South China University of Technology, 382 East Waihuan Rd., Guangzhou Higher Education Mega Centre, Panyu District, Guangzhou 510640, China. 'Key Laboratory of Fermentation Engineering, Ministry of Education, Hubei University of Technology, 1 Lijiadun Rd., Wuhan 430068, China. 


\section{Authors' contributions}

$\mathrm{CH}$ designed and performed the experiments, analyzed the data and wrote the manuscript; HW designed the experiments, analyzed the data and revised the manuscript; ZJL performed several experiments, JC and WYL participated in the design of some experiments, MHZ coordinated the study and revised the manuscript. All authors read and approved the final manuscript.

\section{Competing interests}

The authors declare that they have no competing interests.

Received: 24 October 2011 Accepted: 19 January 2012

Published: 19 January 2012

\section{References}

1. Demirbas A: Progress and recent trends in biofuels. Prog Energy Combust Sci 2007, 33:1-18.

2. Xue FY, Zhang $X$, Luo H, Tan TW: A new method for preparing raw material for biodiesel production. Process Biochem 2006, 41:1699-1702.

3. Adamczak M, Bornscheuer U, Bednarski W: The application of biotechnological methods for the synthesis of biodiesel. Eur J Lipid Sci Technol 2009, 111:800-813.

4. In Single Cell Oil. Edited by: Moreton RS. Harlow, UK: Longman Scientific 1988.

5. Moreton R: Modification of fatty acid composition of lipid accumulating yeasts with cyclopropene fatty acid desaturase inhibitors. Appl Microbiol Biotechnol 1985, 22:42-45.

6. Papanikolaou S, Aggelis G: Yarrowia lipolytica: A model microorganism used for the production of tailor-made lipids. Eur I Lipid Sci Technol 2010, 112:639-654.

7. Wu S, Hu C, Zhao X, Zhao ZB: Production of lipid from Nacetylglucosamine by Cryptococcus curvatus. Eur J Lipid Sci Technol 2010, 112:727-733.

8. Zhao X, Kong XL, Hua YY, Feng B, Zhao ZB: Medium optimization for lipid production through co-fermentation of glucose and xylose by the oleaginous yeast Lipomyces starkeyi. Eur J Lipid Sci Technol 2008, 110:405-412.

9. Li Q, Du W, Liu DH: Perspectives of microbial oils for biodiesel production. Appl Microbiol Biotechnol 2008, 80:749-756

10. Zhu LY, Zong MH, Wu H: Efficient lipid production with Trichosporon fermentans and its use for biodiesel preparation. Bioresour Technol 2008, 99:7881-7885.

11. Papanikolaou S, Aggelis G: Biotechnological valorization of biodiesel derived glycerol waste through production of single cell oil and citric acid by Yarrowia lipolytica. Lipid Technol 2009, 21:83-87.

12. Huang C, Zong MH, Wu H, Liu QP: Microbial oil production from rice straw hydrolysate by Trichosporon fermentans. Bioresour Technol 2009, 100:4535-4538.

13. Reddy N, Yang Y: Biofibers from agricultural byproducts for industrial applications. Trends Biotechnol 2005, 23:22-27.

14. Palmqvist $E_{1}$ Hahn-Hagerdal B: Fermentation of lignocellulosic hydrolysates. II: inhibitors and mechanisms of inhibition. Bioresour Technol 2000, 74:25-33.

15. Almeida J, Modig T, Petersson A, Hahn-Hägerdal B, Lidén G, GorwaGrauslund M: Increased tolerance and conversion of inhibitors in lignocellulosic hydrolysates by Saccharomyces cerevisiae. J Chem Technol Biotechnol 2007, 82:340-349.

16. Chen X, Li Z, Zhang X, Hu F, Ryu D, Bao J: Screening of oleaginous yeast strains tolerant to lignocellulose degradation compounds. Appl Biochem Biotechnol 2009, 159:1-14.

17. Hu C, Zhao X, Zhao J, Wu S, Zhao ZB: Effects of biomass hydrolysis byproducts on oleaginous yeast Rhodosporidium toruloides. Bioresour Technol 2009, 100:4843-4847.

18. Economou CN, Aggelis G, Pavlou S, Vayenas D: Modeling of single-cell oil production under nitrogen-limited and substrate inhibition conditions. Biotechnol Bioeng 2011, 108:1049-55.

19. Mussatto S, Dragone G, Roberto I: Influence of the toxic compounds present in brewer's spent grain hemicellulosic hydrolysate on xylose-toxylitol bioconversion by Candida guilliermondii. Process Biochem 2005, 40:3801-3806.
20. Tran A, Chambers R: Red oak wood derived inhibitors in the ethanol fermentation of xylose by Pichia stipitis CBS 5776. Biotechnol Lett 1985, 7:841-845.

21. Papanikolaou S, Galiotou-Panayotou M, Fakas S, Komaitis M, Aggelis G: Citric acid production by Yarrowia lipolytica cultivated on olive-mill wastewater-based media. Bioresour Technol 2008, 99:2419-2428.

22. Aggelis G, Komaitis M: Enhancement of single cell oil production by Yarrowia lipolytica growing in the presence of Teucrium polium L. aqueous extract. Biotechnol Lett 1999, 21:747-749.

23. Sarris D, Galiotou-Panayotou M, Koutinas AA, Komaitis M, Papanikolaou S: Citric acid, biomass and cellular lipid production by Yarrowia lipolytica strains cultivated on olive mill wastewater-based media. J Chem Technol Biotechnol 2011, 86:1439-1448.

24. Zaldivar J, Ingram LO: Effect of organic acids on the growth and fermentation of ethanologenic Escherichia coli LY01. Biotechnol Bioeng 1999, 66:203-210.

25. Huang C, Wu H, Liu QP, Li YY, Zong MH: Effects of aldehydes on the growth and lipid accumulation of oleaginous yeast Trichosporon fermentans. J Agric Food Chem 2011, 59:4606-4613.

26. Narendranath $\mathrm{N}$, Thomas $\mathrm{K}$, Ingledew W: Effects of acetic acid and lactic acid on the growth of Saccharomyces cerevisiae in a minimal medium. J Ind Microbiol Biotechnol 2001, 26:171-177.

27. Papanikolaou S, Sarantou S, Komaitis M, Aggelis G: Repression of reserve lipid turnover in Cunninghamella echinulata and Mortierella isabellina cultivated in multiple-limited media. J Appl Microbiol 2004, 97:867-875.

28. Ratledge C: Fatty acid biosynthesis in microorganisms being used for single cell oil production. Biochimie 2004, 86:807-815.

29. Wynn JP, Ratledge C: Malic enzyme is a major source of NADPH for lipid accumulation by Aspergillus nidulans. Microbiology 1997, 143:253-257.

30. Papanikolaou S, Gortzi O, Margeli E, Chinou I, Galiotou-Panayotou M, Lalas S: Effect of Citrus essential oil addition upon growth and cellular lipids of Yarrowia lipolytica yeast. Eur J Lipid Sci Technol 2008, 110:997-1006.

31. Aggelis G, Athanassopoulos N, Paliogianni A, Komaitis M: Effect of a Teucrium polium L. extract on the growth and fatty acid composition of Saccharomyces cerevisiae and Yarrowia lipolytica. Antonie van Leeuwenhoek 1998, 73:195-198.

32. Duarte LC, Carvalheiro F, Neves I, Gírio FM: Effects of aliphatic acids, furfural, and phenolic compounds on Debaryomyces hansenii CCMI 941. Appl Biochem Biotechnol 2005, 121:413-425.

33. Oliva J, Negro M, Sáez F, Ballesteros I, Manzanares P, González A, Ballesteros M: Effects of acetic acid, furfural and catechol combinations on ethanol fermentation of Kluyveromyces marxianus. Process Biochem 2006, 41:1223-1228.

34. Oliva JM, Ballesteros I, Negro MJ, Manzanares P, Cabañas A, Ballesteros M: Effect of binary combinations of selected toxic compounds on growth and fermentation of Kluyveromyces marxianus. Biotechnol Progr 2004, 20:715-720.

35. Kavadia A, Komaitis M, Chevalot I, Blanchard F, Marc I, Aggelis G: Lipid and $Y$-linolenic acid accumulation in strains of Zygomycetes growing on glucose. J Am Oil Chem Soc 2001, 78:341-346.

36. Folch J, Lees M, Sloane-Stanley G: A simple method for the isolation and purification of total lipids from animal tissues. J Biol Chem 1957 226:497-509.

doi:10.1186/1754-6834-5-4

Cite this article as: Huang et al:: Effect of organic acids on the growth and lipid accumulation of oleaginous yeast Trichosporon fermentans. Biotechnology for Biofuels 2012 5:4. 